\title{
Proteomic Analysis to Explore the Effect of Zishen Jiangtang Pills on Diabetic Osteoporosis Rats
}

\section{Shufang Chu}

Shenzhen TCM Hospital, the Fourth Clinical Medical College of Guangzhou University of Chinese Medicine

\section{Deliang Liu}

Shenzhen TCM Hospital, the Fourth Clinical Medical College of Guangzhou University of Chinese Medicine

\section{Hengxia Zhao}

Shenzhen TCM Hospital, the Fourth Clinical Medical College of Guangzhou University of Chinese Medicine

\section{Mumin Shao}

Shenzhen TCM Hospital, the Fourth Clinical Medical College of Guangzhou University of Chinese Medicine

\section{Jianping Chen}

Shenzhen TCM Hospital, the Fourth Clinical Medical College of Guangzhou University of Chinese

Medicine

\section{Xuemei Liu}

Shenzhen TCM Hospital, the Fourth Clinical College of Guangzhou University of Chinese Medicine

\section{Xin Qu}

Shenzhen TCM Hospital, the Fourth Clinical Medical College of Guangzhou University of Cinese

Medicine

\section{Zengying Li}

Shenzhen TCM Hospital, the Fourth Clinical Medical College of Guangzhou University of Chinese Medicine

\section{Jinhua Li}

Shenzhen TCM Hospital, the Fourth Clinical Medical College of Guangzhou University of Chinese Medicine

Huilin Li ( $\nabla$ sztcmlhl@163.com )

Shenzhen TCM Hospital, the Fourth Clinical Medical College of Guangzhou University of Chinese Medicine

\section{Research}


Keywords: Diabetes, Diabetes Osteoporosis, Zishen Jiangtang Pill, Proteomic Analysis, Chinese Traditional Medicine, Signal Pathway, Animal Model, Bioinformatic Analysis

Posted Date: August 26th, 2020

DOI: https://doi.org/10.21203/rs.3.rs-63628/v1

License: (c) (1) This work is licensed under a Creative Commons Attribution 4.0 International License. Read Full License 


\section{Abstract}

Context Zishen Jiangtang Pill (ZJP) is a Chinese herbal compound that could play a positive role in the treatment of Diabetic osteoporosis (DOP) by regulating glucose and bone metabolism. However, the specific mechanisms are still unclear.

Background To explore the effect and mechanism of ZJP on DOP rats by proteomic analysis.

Materials and methods After the establishment of diabetes model by $0.2 \% \mathrm{STZ}, 40$ Wistar rats were equally divided into normal group, model group (diabetic rats), high dose group ( $3.0 \mathrm{~g} / \mathrm{kg} / \mathrm{d}$ ZJP) and low dose group $(1.5 \mathrm{~g} / \mathrm{kg} / \mathrm{d}$ ZJP), and received 3 months of treatment. Histological changes in bone and pancreas tissues were observed by Hematoxylin and eosin staining, electron microscopy, and immunofluorescence. Proteomic and bioinformatics analyses were performed to identify the differentially expressed proteins. The fingerprint and active ingredients of ZJP were identified via HPLC.

Results Compared with the model group, ZJP could rescue the weight, fasting blood glucose and fasting insulin of rats in both high and low dose group. ZJP could also improve the micro-structures of pancreatic islet cells, and bone mass, trabecular and marrow cavities in DOP rats. Bioinformatic analysis suggested that ZJP might influence DOP via multiple pathways, mainly including ribosomes, vitamin digestion and absorption, and fat digestion and absorption. The primary active ingredients, including notoginsenoside R1, ginsenoside Rg1, ginsenoside Re, icariin, and ginsenoside Rb1 were detected.

Conclusion ZJP could significantly improve the histomorphology and ultrastructure of bone and islets tissues, and might serve as an effective alternative medicine for the treatment of DOP.

\section{Background}

Diabetes mellitus (DM) is a group of metabolic diseases characterized by high blood glucose that pose a serious danger towards human health. According to prior reports, about $10 \%$ of adults worldwide have diabetes; moreover, the incidence of diabetes is growing at an alarming rate (Mannino et al. 2018). Complications from diabetes mainly involve the kidneys, retinas, nervous system, and cardiovascular system. Additionally, a recent epidemiological study suggests that the skeletal system is also an important target of diabetes-mediated damage (Rubin 2017; Schacter and Leslie 2017).

Diabetic osteoporosis (DOP) is a common diabetic complication (Hamann et al. 2012; Lechleitner et al. 2013). The main pathological changes associated with DOP are a decrease in bone mass per unit volume, a reduction in bone strength, and an increase in bone fragility (Kurra and Siris 2011; Montagnani et al. 2011). With the aging of the global population, the incidence of DM is increasing every year, which directly leads to an increase in the incidence of DOP (Vestergaard 2007; Montagnani and Gonnelli 2013). At present, the treatment of DOP is mainly based on anti-osteoporosis drugs (Schwartz 2017; Wang T et al. 2017). Western medicine treatments generally include taking calcium, vitamin $D$, sex hormones, bisphosphonates, calcitonin, and/or fluoride or using monoclonal antibodies(Chang et al. 2018; Gallagher 
2018; Suzuki T et al. 2018). However, the therapeutic effects of such drugs are still controversial, and some patients cannot tolerate their side effects or afford their expensive prices. As such, there is an urgent need to identify the mechanism of osteoporosis in patients with diabetes in an effort to identify more cost-effective treatments.

Recently, a large number of experimental and clinical studies detailing the treatment of DOP have affirmed the efficacy of Traditional Chinese Medicine (TCM)(Choi et al. 2012; Qi et al. 2013; Li XJ et al. 2016). Zishen Jiangtang Pill (ZJP) is a TCM compound developed by Shenzhen TCM Hospital. The components of ZJP have the functions of nourishing the breath feminine, nourishing the kidneys, and strengthening the bones. Its use is in line with the theory of "kidney dominating bone" for the treatment of osteoporosis. Previous study have found that ZJP has a positive function in regard to hypoglycemic action, decreasing lipids, and improving insulin sensitivity, and revealed that ZJP could play an antiosteoporosis role on multi-targets, as based on in vitro experiments( $\mathrm{Li} \mathrm{H}$ et al. 2018). However, the molecular mechanism by which ZJP works is still not fully understood.

We established a rat model of DOP and treated these rats with different doses of ZJP. We then observed the histomorphology of bone and pancreatic tissues. Gene ontology (GO) and Kyoto Encyclopedia of Genes and Genomes (KEGG)analyses were also employed to explore the effects of ZJP in these DOP rats and to explore the molecular mechanisms. High-performance liquid chromatography (HPLC) finger print was applied to identify active ingredients of ZJP.

\section{Materials And Methods}

\section{Drugs, reagents, and antibodies}

Zishen Jiangtang Pills (ZJP) was prepared by the Pharmacy Department of Shenzhen TCM Hospital. The main components were Astragalus membranaceus (Astragalus Linn.), Codonopsis pilosula (Codonopsis Wall.), Carapax Trionycis (Trionychidae), Panax Notoginseng (Panax L.), Rehmannia glutinosa (Rehmannia), Schisandra chinensis (Schisandra Michx.), Polygonatum (Liliaceae), Achyranthes bidentata (Achyranthes L), Ostrea gigas tnunb (Ostrea), Rhizoma Drynariae (Davalliaceae) (Batch No: Guangdong Z20070085). This mixture was dissolved with warm distilled water and concentrated to a crude drug containing $1 \mathrm{~g} / \mathrm{mL}$.

The main reagents and antibodies used are as follows: Streptozocin (STZ) and carboxymethyl cellulose sodium (CMC) (No. S0130/C4888, Sigma, USA). Insulin primary antibody (No.3014s, CST, USA), fluorescent secondary antibody (No.111-585-003, Jackson Immuno Research Laboratories, inc, USA; No. A23210, Abbkine, USA), glucagon primary antibody (No.ab10988, Abcam, UK), Dapi fluorescent seal tablets (No.0100 - 20, Southern Biotech), chromatographically pure acetonitrile (Merck, USA), and the reference products including notoginsenoside R1, ginsenoside Rg1, ginsenoside Re, icariin, and ginsenoside Rb1 (China Food and Drug Testing Institute).

\section{Animals}


A total of 70 2-month-old SPF male Wistar rats, weighing from 160 to $190 \mathrm{~g}$, were purchased from the experimental animal center of Southern Medical University. The animals were housed in the SPF room in Shenzhen Municipal Center for Disease Control and Prevention, with an ambient temperature of 18$22^{\circ} \mathrm{C}$, natural circadian rhythm illumination ( $12 \mathrm{~h}: 12 \mathrm{~h}$ ), and an environmental humidity of $40-70 \%$. All animal experiments were in accordance with the Animal Research Ethics Committee and approved by the Animal Protection and Use Committee of the Guangdong Experimental Animal Center.

\section{Establishment of a rat diabetes model}

Rats were adaptively fed for 2 weeks, and 10 rats were randomly selected as normal controls. The remaining 60 rats were used to establish the diabetes model. After fasting for $12 \mathrm{~h}$, rats were injected intraperitoneally with $0.2 \%$ STZ (diluent $0.1 \mathrm{M}$ citrate-sodium buffer, $\mathrm{pH} 4.5$ ) at a dose of $60 \mathrm{mg} / \mathrm{kg}$. The blood glucose of the rats was measured using a blood glucose meter $72 \mathrm{~h}$ after administration. Rats with a fasting blood glucose $>16.7 \mathrm{mmol} / \mathrm{L}$ were fed commonly for 2 weeks. Those rats that still had a fasting blood glucose $>16.7 \mathrm{mmol} / \mathrm{L}$ were considered as successful models. The normal control group was injected intraperitoneally with the same amount of $0.1 \mathrm{M}$ citrate-sodium buffer $(\mathrm{pH}=4.5)$. Some rats were failed or dead in the establishment of diabetes model. Finally, a total of four groups with 10 rats in each group were obtained. The rats of four groups then received a 3-month treatment. The normal group and model group were given $0.5 \%$ sodium carboxymethyl cellulose $10 \mathrm{~mL} / \mathrm{kg} /$ day via intragastric administration, and the high ( $3.0 \mathrm{~g} / \mathrm{kg} / \mathrm{d}$ ZJP) and low dose $(1.5 \mathrm{~g} / \mathrm{kg} / \mathrm{d}$ ZJP) groups were simultaneously given with $0.5 \%$ sodium carboxymethyl cellulose $10 \mathrm{~mL} / \mathrm{kg} /$ day via intragastric administration. At the end of the treatment, all of the rats were fasted for $12 \mathrm{~h}$ and then anesthetized using sodium pentobarbital $(50 \mathrm{mg} / \mathrm{kg}$ ) and sacrificed via abdominal aorta bleeding. The blood was obtained, and the pancreas and bone tissues were stored at $-80^{\circ} \mathrm{C}$. All animal experiments were in accordance with the Animal Research Ethics Committee and approved by the Animal Protection and Use Committee of the Guangdong Experimental Animal Center.

\section{Hematoxylin and eosin (H\&E) staining}

Pancreatic tissues were sliced, fixed in $4 \%$ paraformaldehyde, rinsed with water for $24 \mathrm{~h}$, dehydrated with an ethanol gradient, placed in anhydrous acetone, and embedded in polymethyl methacrylate. H\&E staining was performed as follows: xylene for $5 \mathrm{~min}$, xylene for $3 \mathrm{~min}, 100 \%$ alcohol for $30 \mathrm{~s}, 100 \%$ alcohol for $30 \mathrm{~s}, 95 \%$ alcohol for $30 \mathrm{~s}, 90 \%$ alcohol for $30 \mathrm{~s}$, hematoxylin staining for $10-15 \mathrm{~min}, 1 \%$ hydrochloric acid alcohol differentiated slice for $10 \mathrm{~s}, 1 \%$ eosin staining for $3 \mathrm{~min}, 90 \%$ alcohol for $30 \mathrm{~s}$, $95 \%$ alcohol for $30 \mathrm{~s}(2 \mathrm{x}), 100 \%$ alcohol for $30 \mathrm{~s}(3 \mathrm{x})$, carbonic acid xylene for $30 \mathrm{~s}$, xylene for $30 \mathrm{~s}(3 \mathrm{x})$, and a neutral gum seal.

\section{Double-label pancreatic immunofluorescence}

The paraffin slices were baked at $60^{\circ} \mathrm{C}$, dewaxed with xylene and ethanol, and repaired using citrate antigen repair solution. The slices were then incubated with insulin (1:400) and glucagon (1:1000) primary antibodies in the dark at $4^{\circ} \mathrm{C}$ overnight. The slices were then incubated with a fluorescent secondary antibody $(1: 100)$ at $37^{\circ} \mathrm{C}$ in the dark for $1 \mathrm{~h}$, stained with DAPI, and sealed using a fluorescent 
sealing tablet. The positive islet beta cells appeared red, the positive islet alpha cells appeared green, and the nuclei appeared blue.

\section{Pancreatic transmission electron microscopy}

Pancreatic tissues were fixed with $2.5 \%$ glutaraldehyde, dehydrated in ethanol, penetrated with propylene oxide, embedded with a 1:1 propylene oxide and resin mixture for $2 \mathrm{~h}$, pure resin for $2 \mathrm{~h}$, and resin at $48^{\circ} \mathrm{C}$ for $10 \mathrm{~h}$. The samples were then sliced at 500-1000 nm, stained with toluidine blue, sliced at $70 \mathrm{~nm}$, and stained with lead and uranium.

\section{Femoral electron microscope scanning}

The fixed bone tissue sections were processed as follows: sections were stained with the Weigert's iron lignin on a glass slide for 40 min and rinsed with water until it turned blue. These were then incubated in $1 \%$ hydrochloric acid ethanol and washed with water until it turned blue, dyed with Van Gieson picric acidmagenta solution for 3 min, dehydrated with 95\% ethanol and anhydrous ethanol, quickly dipped in fresh anhydrous ethanol, and then blotted dry. After the above treatment, the sections were immersed in an acetonitrile solution, which was then replaced with $70 \%, 80 \%, 90 \%, 95 \%, 100 \%$ acetonitrile, soaked for $15-$ 20 min each time, and finally replaced with $100 \%$ acetonitrile and dried. The sections were then sprayed with carbon and gold and observed under an electron microscope.

\section{Proteomics analysis}

The proteins were extracted from the rats bone tissues in different groups. The extracted protein samples were subjected to reductive alkylation treatment to open the disulfide bonds for subsequent enzymatic hydrolysis of the proteins. Trypsin and 8-plex iTRAQ reagent (AB Sciex, Cat. No.4381664) were used to label the protein. The mixed peptides were pre-isolated using high $\mathrm{pH}$ reverse phase chromatography (High pH-RP Chromatography), and liquid chromatography was performed coupled with tandem mass spectrometry (LC-MS/MS) analysis. The mass spectrometry data was assessed using Protein Pilot software (AB, Version 5.0) and aligned for identification; the database used was UniProtKB/Swiss-Prot. The identification criteria for the differentially expressed proteins was a fold difference of $\geq 1.5$ or $\leq$ 0.667 , and the number of unique peptides per protein $\geq 2$ and $<0.05$ was considered to be a significant difference.

The Omicsbean(http://www.omicsbean.cn/) multi-functional bioinformatics analysis tool, integrated STRING (http://www.string-db.org) biological database, and Cytoscape software were used to perform enrichment analysis on the identified differentially expressed proteins based on GO biological process, cellular component, and molecular function. Additionally, KEGG (http://www.kegg.jp/kegg/pathway.html) biological pathway enrichment analysis was performed on the differentially expressed proteins.

\section{HPLC fingerprinting and active ingredient detection}

A reference solution that contained notoginsenoside R1, ginsenoside Rg1, ginsenoside Re, icariin, and ginsenoside Rb1 was mixed with methanol at a concentration of $0.1 \mathrm{mg} / \mathrm{mL}$. The ZJP powder was 
dissolved in $100 \%$ methanol and filtered as test solution. The reference solution and the test solution were tested via HPLC, and the characteristic maps of both solutions were obtained. The chromatographic conditions were as follows: octadecyl silane-bonded silica gel was used as filler, and a gradient elution was carried out at a detection wavelength of $203 \mathrm{~nm}$. The column was an Agilent TC-C18 $(250 \mathrm{~mm} \times$ $4.6 \mathrm{~mm}, 5 \mu \mathrm{m}$ ) with water as mobile phase A and acetonitrile as mobile phase B. Gradient elution: 0$12 \mathrm{~min}, 81 \% \mathrm{~A}, 12-60 \mathrm{~min}, 81 \%-64 \% \mathrm{~A}$, flow rate $1.0 \mathrm{~mL} / \mathrm{min}$, and a detection wavelength of $203 \mathrm{~nm}$.

\section{Statistical Analysis}

The obtained data were statistically analyzed and processed using State12.0 software, and the measured data were expressed as the mean \pm standard deviation ( \pm SD). One-way analysis of variance (ANOVA) was used to assess the differences between the groups, and the Bonferroni test was used for multiple comparisons between the groups. Wilcoxon rank sum test was used for comparison between the group data that did not conform to a normal distribution or variance. Statistical significance was defined as $P<$ 0.05 .

\section{Results}

\section{Body weight, fasting blood glucose and fasting insulins}

The body weight of the model group $(238.7 \pm 22.08 \mathrm{~g})$ was significantly lower than that in the normal group $(408.8 \pm 21.06 \mathrm{~g})(\mathrm{P}<0.01)$. Compared with the model group, the rats in the high-dose ZJP group $(308.3 \pm 19.35 \mathrm{~g})$ and the low-dose ZJP group $(305.9 \pm 13.25 \mathrm{~g})$ had gained significant weight $(\mathrm{P}<$ 0.01). The results are shown in Fig. 1A. In addition, as shown in Fig. 1B, both the high-dose (13.16 \pm $2.15 \mathrm{mM})$ and low-dose groups $(14.66 \pm 1.22 \mathrm{mM})$ of ZJP could effectively reduce the fasting blood glucose of the model group $(23.79 \pm 5.54 \mathrm{mM})(P<0.01)$, and there was no significant difference between the two groups. Also, ZJP could significant improve the fasting insulin of model group(514.0 \pm $180.7 \mathrm{pg} / \mathrm{mL})(P<0.01)$, while there no significant difference between high-dose $(2360 \pm 718.2 \mathrm{pg} / \mathrm{mL})$ and low-dose group(2508 $\pm 925 \mathrm{pg} / \mathrm{mL}$ ) (Fig. 1C).

\section{ZJP can improve the status of pancreatic islet cells in DOP model rats}

We assessed the effect of ZJP on pancreatic islet cells by H\&E staining, immunofluorescence and transmission electron microscopy. The islet cells in the normal group were lightly stained, with a high cell density, uniform distribution, and regular morphology (Fig. 2A, E). The mitochondria, endoplasmic reticulum, and Golgi were abundant in the cytoplasm of the normal group (Fig. 2I). In the model group, the islets were significantly reduced, their shape was irregular, their cell density was reduced, and more cells presented with nuclear pyknosis. The peripheral acinar cells also demonstrated substantial atrophy and degeneration (Fig. 2B, F). Moreover, the number of mitochondria was decreased, and they were swollen and deformed, the endoplasmic reticulum had expanded to different extents, and a degranulation phenomenon could be seen (Fig. 2J). The pancreatic islet structures in the high-dose and low-dose ZJP 
groups were significantly improved compared with the model group. In these treatment groups, the islet size was basically normal, the morphology was regular, the islet cells were arranged neatly, the density was acceptable, and the number of atrophied and degenerated islet cells were decreased (Fig. 2C, D, G and $\mathrm{H}$ ). Additionally, in these treatment groups, the endoplasmic reticulum were not significantly expanded (Fig. 2K, L).

\section{ZJP can improve the histological morphology of bone tissues in DOP model rats}

In addition to the effects of bone mass reduction, DOP is also related to bone structure factors and the number of micro-injuries in the bone(An YH et al. 1997; Coe et al. 2011). Therefore, we performed electron microscopy to assess for these changes in the bone microstructures. The bone density of the femurs in the normal group was thick and, the trabecular bones were arranged neatly. The surface of the trabecular bone was regular and smooth, the gap between the trabeculae was small, and the collagen fibers were neatly arranged in the trabecular bone (Fig. 3A). In the model group, the bone density appeared significantly thinner, the marrow cavity was larger, the trabecular bone connection was interrupted, and the reticular structure was destroyed (Fig. 3B). The trabecular bone structures in the high and low dose ZJP groups were more organized compared with the model group. The bone marrow cavities were also smaller (Fig. 3C, D).

\section{GO and KEGG analysis of ZJP function in DOP model rats}

In order to better clarify how ZJP exerts its anti-osteoporosis function, we carried out proteomic GO and KEGG analyses. We classified the altered proteins after low-dose ZJP treatment into GO classification due to the equal effect with high-dose ZJP treatment on DOP rats. Comparing to the normal group, the results in the model group indicated that diabetic osteoporosis mostly resulted in protein changes in biological process including anatomical structure development $(13 \%)$, response to stress $(13 \%)$, biosynthetic process (12\%), and cellular nitrogen compound metabolic process(12\%) (Fig. 4A). The molecular functions of these proteins most likely reflected ion binding (19\%), RNA binding (17\%), and enzyme binding (15\%) (Fig. 4B). As for the cellular component, the highest probability of existence included the extracellular region (17\%), organelle (17\%), and cytoplasm (16\%) (Fig. 4C). By comparing the low-dose ZJP group with the model group, we arrived at a similar conclusion in regard to these three categories (Fig. 4D-F).

We then performed pathway enrichment analysis on the differentially expressed proteins. Comparing the normal group with the model group, the pathways with the highest degree of enrichment involved the following aspects: fatty acid elongation, arginine and proline metabolism, fatty acid degradation, PPAR signaling pathway, fat digestion and absorption, adipocytokine signaling pathway, and DNA replication (Fig. 5A). While comparing the model group with ZJP treatment groups, the most enriched pathways were ribosome, vitamin digestion and absorption, DNA replication, arginine and proline metabolism, and fat digestion and absorption (Fig. 5B).

\section{ZJP Fingerprint and Active Ingredients}


We first determined the fingerprint map of ZJP via HPLC and identified its active ingredients (Fig. 6). There were five major characteristic peaks in the map with relative retention time specifications, including notoginsenoside R1 (peak 1), ginsenoside Rg1 (peak 2), ginsenoside Re (peak 3), icariin (peak 4), and ginsenoside Rb1 (peak 5).

\section{Discussion}

DOP is a common complication of DM and is characterized by low bone mass and micro-structural degradation of bone tissue leading to increased bone fragility. With the aging of the global population, the incidence of both DM and DOP is becoming higher and affecting people's quality of life. At present, the treatment for DOP is similar to that for osteoporosis and includes three types of treatments: supplementation of calcium and vitamin D(Ross et al. 2011; Li K et al. 2012), inhibition of bone resorption with drugs such as bisphosphonates(Coe et al. 2015; Davis et al. 2016; Takeuchi 2017), and promotion of bone formation with drugs such as PTH1-34(Tsuchida et al. 2000; Suzuki K et al. 2003). However, the overall effect of these treatments is not particularly good, with a high rate of side effects and a high cost. Currently, numerous studies are focusing on the effects of TCM in regard to osteoporosis(Ju et al. 2014; Li Y et al. 2014; Lai et al. 2015), but the concise role and mechanism underlying such treatments are still unclear. As such, it is important to understand the molecular mechanism and related pathogenesis of DOP, so as to help identify an effective therapy. Moreover, it is necessary to detail the targets, holistic mechanism, compound compatibility, and safety of TCM compounds using genomic technologies, which will strongly promote the pace of modern research regarding Chinese medicinal compounds.

Previous study has demonstrated that ZJP can effectively improve glucose metabolism and abnormal bone metabolism, and regulate blood and urinary metabolism in DOP rats( $\mathrm{Li} \mathrm{H}$ et al. 2018). ZJP has a clear hypoglycemic and lipid-lowering effect, can improve insulin sensitivity, promote resistance to oxidation, protect the vascular endothelium, and reduce the level of inflammatory factors(Yunshan et al. 2008; Yongping et al. 2012). Bases on the establishment of our DOP rat model, we found that ZJP could reduce the weight loss of rat caused by DOP, improve the fasting blood glucose and fasting insulin of these rats (Fig. 1). The most important changes occurring in patients with DOP involve alterations in blood glucose and the skeletal system. Therefore, microscopic analyses of islet cells and bone tissues were performed in the present study. The results indicated that ZJP conferred an obvious improvement to the histomorphology and ultrastructure of islets (Fig. 2) and could improve bone formation, reduce bone resorption, increase bone density, and improve the bone microstructure (Fig. 3). These pathology results support our previous findings( $\mathrm{Li} \mathrm{H}$ et al. 2018) that ZJP was shown to exert its influence by affecting aspects of glucose metabolism and bone metabolism.

To further investigate the potential mechanism of ZJP in the treatment of DOP, we performed proteomic GO and KEGG analyses on the identified differentially expressed proteins. The results revealed that ZJP most likely affected ion binding, RNA binding, and enzyme binding involved in several biological processes, including anatomical structure development, response to stress, biosynthetic processes, and 
cellular nitrogen compound metabolic processes. The roles of ZJP in these aspects are closely related to known factors related to the occurrence and development of DOP. Moreover, these roles are reflected in several aspects of DOP, such as bone structure changes, blood metabolism, and others. The highest probability of existence in regard to the cellular component were the extracellular region, organelles, and cytoplasm, indicating that it is possible that ZJP might have some functions affecting the cellular microenvironment. The enrichment pathways identified mostly referred to the ribosome, vitamin digestion and absorption, DNA replication, arginine and proline metabolism, and fat digestion and absorption, indicating that ZJP is closely related to changes in body weight and calcium absorption as well as protein synthesis. However, to further characterize the protein signaling network associated with the treatment of DOP via ZJP, it will be necessary to further identify the specific target genes via additional methods, both in vitro and in vivo, in an effort to define the mechanism of action of ZJP in regard to the treatment of DOP.

ZJP is a Chinese herbal compound developed by Shenzhen TCM hospital, and this study was the first to define the fingerprint map of ZJP via HPLC and ascertain the primary active ingredients, including notoginsenoside R1, ginsenosideRg1, ginsenoside Re, icariin, and ginsenoside Rb1. Thus, notoginsenoside R1, ginsenoside Rg1, ginsenoside Re, icariin, and ginsenoside Rb1 together were found to have both an anti-hypoglycemic action and anti-osteoporosis effect in the previous study (Shindel et al. 2010; Yu et al. 2015). Pharmacological studies have confirmed that epimedium extracts can inhibit the activity of osteoclasts via various means and promote the differentiation of osteoblasts and increased bone formation and bone density (An J et al. 2016; Wang J et al. 2016; Liu et al. 2018). Ginsenoside Rg1 treatment of diabetic rats was associated with reduced oxidative stress and attenuated myocardial apoptosis (Yu et al. 2015). Icariin could significantly regulatethe nNOS and calponin in penile tissues of all rats (Shindel et al. 2010). Icariin attenuates titanium-particle inhibition of bone formation by activating the Wnt/ $\beta$-catenin signaling pathway in vivo and in vitro(Wang J et al. 2016). These observations provided theoretical support for the prevention and treatment of DOP by ZJP.

\section{Conclusions}

Overall, this study identified the main active components of ZJP and determined that ZJP could significantly improve the histomorphology and ultrastructure of bone and islets tissues using a DOP rat model. The proteomics analysis suggested the possible protein networks and biological roles of ZJP in the treatment of DOP, which highlight its potential mechanisms of action. In the further study, we will focus on one compound as a representative ingredient, and verify the predicted mechanisms.

\section{Declarations}

\section{Ethics approval and consent to participate}

This manuscript was in accordance with the Animal Research Ethics Committee and approved by the Animal Protection and Use Committee of the Guangdong Experimental Animal Center. 


\section{Consent for publication}

This manuscript is approved by all authors for publication.

\section{Availability of data and material}

We declare that all the data and materials of this manuscript are available.

\section{Competing interests}

The authors declared that they have no conflicts of interest to this work.

\section{Funding}

The study was supported by Shenzhen Municipal Science and Technology Bureau (JCYJ20140408153331810, JCYJ20170817094838619); Sanming Project of Medicine in Shenzhen (SZSM201512043).

\section{Authors' contributions}

Chu SF and Li HL designed the study. Chu SF, Liu DL, Zhao HX, Shao MM, Chen JP, Liu XM and Qu X performed the research. Liu DL, Li ZY and Li JH analyzed data. Chu SF and Li HL wrote the paper.

\section{Acknowledgements}

None

\section{References}

1. An J, Yang H, Zhang Q, Liu C, Zhao J, Zhang L, Chen B. 2016. Natural products for treatment of osteoporosis: The effects and mechanisms on promoting osteoblast-mediated bone formation. Life Sci. 147:46-58.

2. An YH, Zhang J, Kang Q, Friedman RJ. 1997. Mechanical properties of rat epiphyseal cancellous bones studied by indentation testing. J Mater Sci Mater Med. 8(8):493-495.

3. Chang B, Quan Q, Li Y, Qiu H, Peng J, Gu Y. 2018. Treatment of osteoporosis, with a focus on 2 monoclonal antibodies. Med Sci Monit. 24:8758-8766.

4. Choi C, Lee H, Lim H, Park S, Lee J, Do S. 2012. Effect of Rubus coreanus extracts on diabetic osteoporosis by simultaneous regulation of osteoblasts and osteoclasts. Menopause. 19(9):10431051.

5. Coe LM, Irwin R, Lippner D, McCabe LR. 2011. The bone marrow microenvironment contributes to type I diabetes induced osteoblast death. J Cell Physiol. 226(2):477-483.

6. Coe LM, Tekalur SA, Shu Y, Baumann MJ, McCabe LR. 2015. Bisphosphonate treatment of type I diabetic mice prevents early bone loss but accentuates suppression of bone formation. J Cell 
Physiol. 230(8):1944-1953.

7. Davis S, Martyn-St James M, Sanderson J, Stevens J, Goka E, Rawdin A, Sadler S, Wong R, Campbell F, Stevenson M, et al. 2016. A systematic review and economic evaluation of bisphosphonates for the prevention of fragility fractures. Health Technol Assess. 20(78):1-406.

8. Gallagher JC. 2018. Advances in osteoporosis from 1970 to 2018. Menopause. 25(12):1403-1417.

9. Hamann C, Kirschner S, Gunther KP, Hofbauer LC. 2012. Bone, sweet bone--osteoporotic fractures in diabetes mellitus. Nat Rev Endocrinol. 8(5):297-305.

10. Ju D, Liu M, Zhao H, Wang J. 2014. Mechanisms of "kidney governing bones" theory in traditional Chinese medicine. Front Med. 8(3):389-393.

11. Kurra S, Siris E. 2011. Diabetes and bone health: the relationship between diabetes and osteoporosisassociated fractures. Diabetes Metab Res Rev. 27(5):430-435.

12. Lai N, Zhang Z, Wang B, Miao X, Guo Y, Yao C, Wang Z, Wang L, Ma R, Li X, et al. 2015. Regulatory effect of traditional Chinese medicinal formula Zuo-Gui-Wan on the Th17/Treg paradigm in mice with bone loss induced by estrogen deficiency. J Ethnopharmacol. 166:228-239.

13. Lechleitner M, Pils K, Roller-Wirnsberger R, Beubler E, Gasser R, Mrak P, Hoppichler F, Pietschmann P. 2013. [Diabetes and osteoporosis: pathophysiological interactions and clinical importance for geriatric patients]. Z Gerontol Geriatr. 46(5):390-397.

14. Li H, Chu S, Zhao H, Liu D, Liu X, Qu X, Chen J, Li Z, Li J. 2018. Effect of Zishen Jiangtang pill, a Chinese herbal product, on rats with diabetic osteoporosis. Evidence-based complementary and alternative medicine : eCAM. 2018:7201914.

15. Li K, Kaaks R, Linseisen J, Rohrmann S. 2012. Associations of dietary calcium intake and calcium supplementation with myocardial infarction and stroke risk and overall cardiovascular mortality in the Heidelberg cohort of the European Prospective Investigation into Cancer and Nutrition study (EPIC-Heidelberg). Heart. 98(12):920-925.

16. Li XJ, Zhu Z, Han SL, Zhang ZL. 2016. Bergapten exerts inhibitory effects on diabetes-related osteoporosis via the regulation of the PI3K/AKT, JNK/MAPK and NF-kappaB signaling pathways in osteoprotegerin knockout mice. Int J Mol Med. 38(6):1661-1672.

17. Li Y, Lu SS, Tang GY, Hou M, Tang Q, Zhang XN, Chen WH, Chen G, Xue Q, Zhang CC, et al. 2014. Effect of Morinda officinalis capsule on osteoporosis in ovariectomized rats. Chin J Nat Med. 12(3):204-212.

18. Liu S, Dong H, Dai H, Liu D, Wang Z. 2018. MicroRNA-216b regulated proliferation and invasion of non-small cell lung cancer by targeting SOX9. Oncology Lletters. 15(6):10077-10083.

19. Mannino GC, Andreozzi F, Sesti G. 2018. Pharmacogenetics of type 2 diabetes mellitus, the route toward tailored medicine. Diabetes Metab Res Rev.e3109.

20. Montagnani A, Gonnelli S. 2013. Antidiabetic therapy effects on bone metabolism and fracture risk. Diabetes Obes Metab. 15(9):784-791. 
21. Montagnani A, Gonnelli S, Alessandri M, Nuti R. 2011. Osteoporosis and risk of fracture in patients with diabetes: an update. Aging Clin Exp Res. 23(2):84-90.

22. Qi W, Zhang Y, Yan YB, Lei W, Wu ZX, Liu N, Liu S, Shi L, Fan Y. 2013. The protective effect of cordymin, a peptide purified from the medicinal mushroom cordyceps sinensis, on diabetic osteopenia in alloxan-induced diabetic rats. Evid Based Complement Alternat Med. 2013:985636.

23. Ross AC, Manson JE, Abrams SA, Aloia JF, Brannon PM, Clinton SK, Durazo-Arvizu RA, Gallagher JC, Gallo RL, Jones $\mathrm{G}$, et al. 2011. The 2011 report on dietary reference intakes for calcium and vitamin D from the Institute of Medicine: what clinicians need to know. J Clin Endocrinol Metab. 96(1):53-58.

24. Rubin MR. 2017. Skeletal fragility in diabetes. Annals of the New York Academy of Sciences. 1402(1):18-30.

25. Schacter GI, Leslie WD. 2017. Diabetes and bone disease. Endocrinology and metabolism clinics of North America. 46(1):63-85.

26. Schwartz AV. 2017. Efficacy of osteoporosis therapies in diabetic patients. Calcif Tissue Int. 100(2):165-173.

27. Shindel AW, Xin ZC, Lin G, Fandel TM, Huang YC, Banie L, Breyer BN, Garcia MM, Lin CS, Lue TF. 2010. Erectogenic and neurotrophic effects of icariin, a purified extract of horny goat weed (Epimedium spp.) in vitro and in vivo. The journal of sexual medicine. 7(4 Pt 1):1518-1528.

28. Suzuki K, Miyakoshi N, Tsuchida T, Kasukawa Y, Sato K, Itoi E. 2003. Effects of combined treatment of insulin and human parathyroid hormone(1-34) on cancellous bone mass and structure in streptozotocin-induced diabetic rats. Bone. 33(1):108-114.

29. Suzuki T, Nakamura Y, Kato H. 2018. Vitamin D and calcium addition during denosumab therapy over a period of four years significantly improves lumbar bone mineral density in Japanese osteoporosis patients. Nutrients. 10(3):272.

30. Takeuchi Y. 2017. [Innovation of bisphosphonates for improvement of adherence]. Clin Calcium. 27(2):197-202.

31. Tsuchida T, Sato K, Miyakoshi N, Abe T, Kudo T, Tamura Y, Kasukawa Y, Suzuki K. 2000. Histomorphometric evaluation of the recovering effect of human parathyroid hormone (1-34) on bone structure and turnover in streptozotocin-induced diabetic rats. Calcif Tissue Int. 66(3):229-233.

32. Vestergaard P. 2007. Discrepancies in bone mineral density and fracture risk in patients with type 1 and type 2 diabetes-a meta-analysis. Osteoporos Int. 18(4):427-444.

33. Wang J, Tao Y, Ping Z, Zhang W, Hu X, Wang Y, Wang L, Shi J, Wu X, Yang H, et al. 2016. Icariin attenuates titanium-particle inhibition of bone formation by activating the Wnt/beta-catenin signaling pathway in vivo and in vitro. Scientific reports. 6:23827.

34. Wang T, Cai L, Wang Y, Wang Q, Lu D, Chen H, Ying X. 2017. The protective effects of silibinin in the treatment of streptozotocin-induced diabetic osteoporosis in rats. Biomed Pharmacother. 89:681688.

35. Yongping X, Bin G, Yingrong L. 2012. Effect of Zishen Jiangtang Pill on insulin sensitivity in figh-fat mouse model and its mechanism. Chinese Medicine Guide. 10(13):7-9. 
36. Yu HT, Zhen J, Pang B, Gu JN, Wu SS. 2015. Ginsenoside Rg1 ameliorates oxidative stress and myocardial apoptosis in streptozotocin-induced diabetic rats. Journal of Zhejiang University Science B. 16(5):344-354.

37. Yunshan G, Huilin L, Hengxia Z. 2008. Effects of Zishen Jiangtang pill on serum adiponectin and vascular endothelial function in patients with diabetic macroangiopathy. China Medical Herald. 5(6):18-20.

Figures
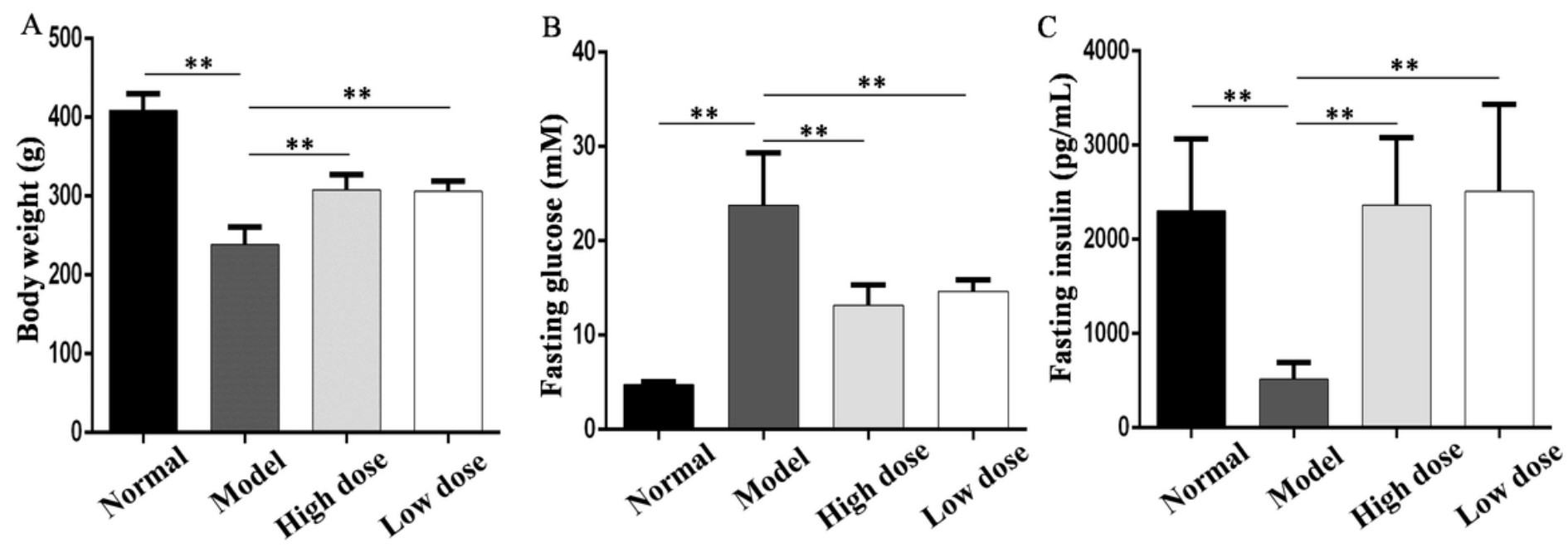

Figure 1

Body weight (A), fasting blood glucose (B) and fasting insulin (C) of each group. 

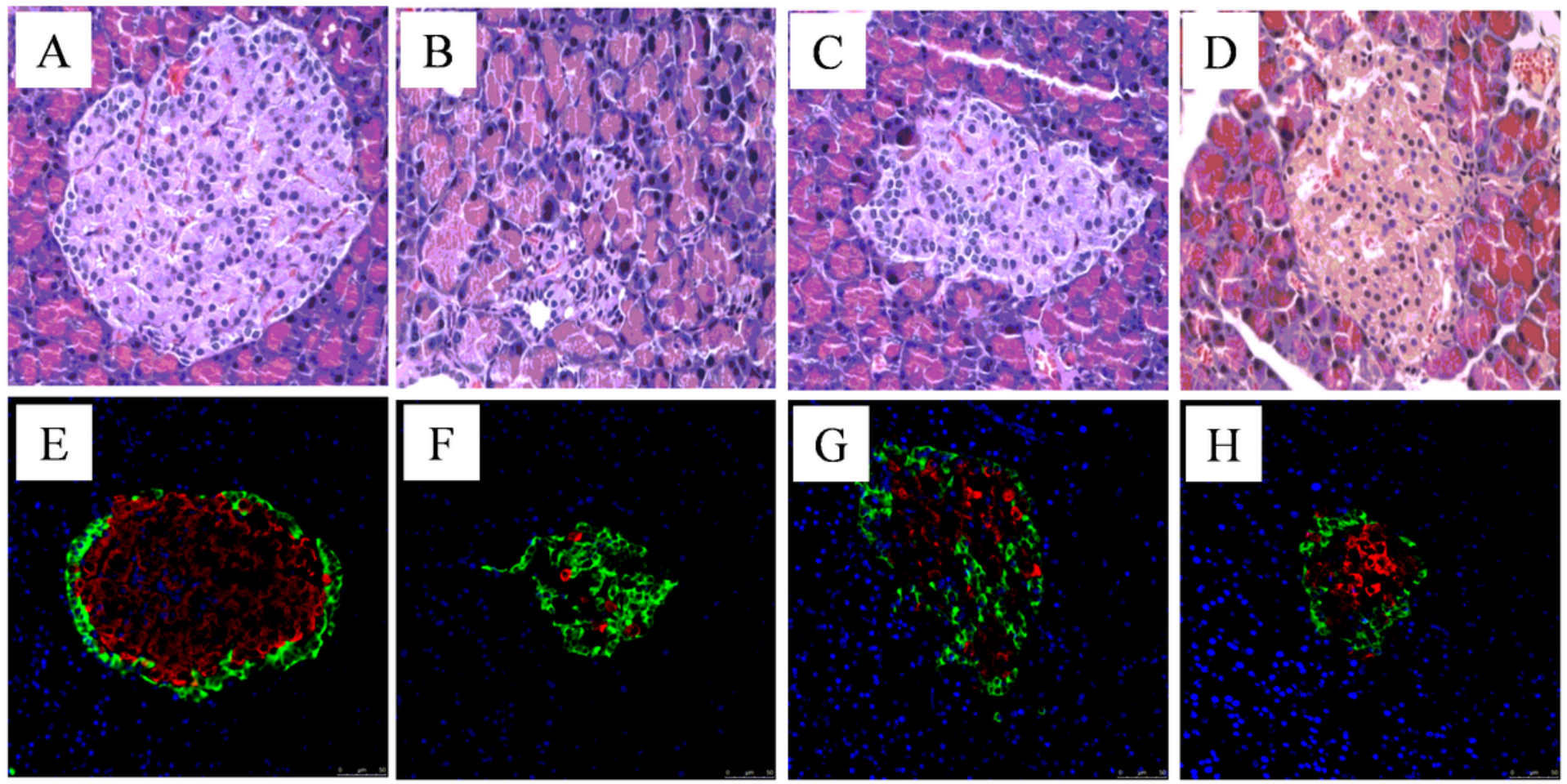

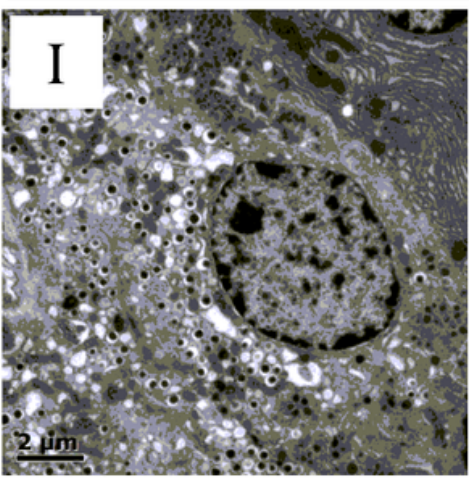

Normal

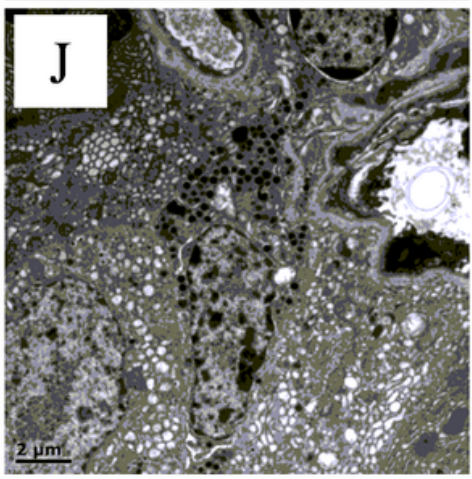

Model

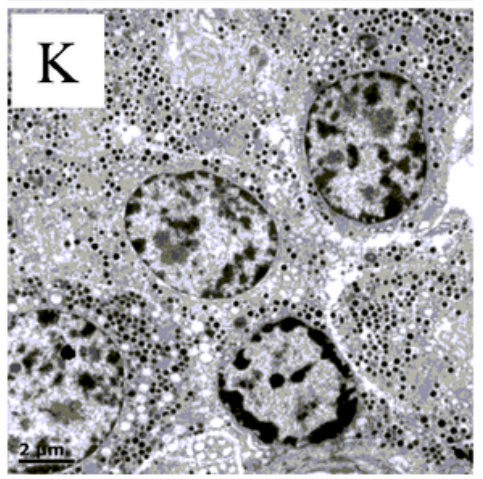

High dose

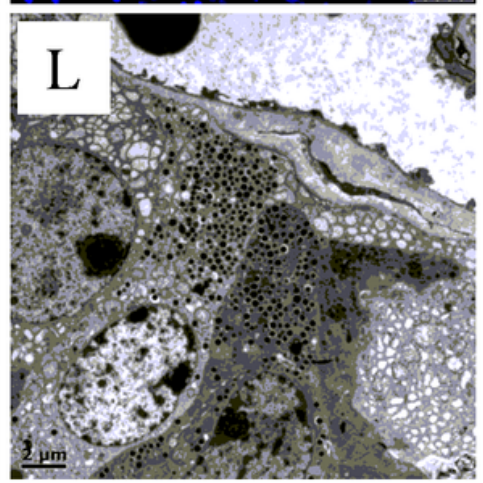

Low dose

Figure 2

Pancreatic microstructure in each group. A-D: H\&E staining ( $\times 400)$; E-H: insulin-glucagon double-label immunofluorescence $(\times 400)$; I-L: Transmission electron microscopy $(\times 5000)$. A, E, I: Normal group. B, F, J: Model group. C, G, K: High-dose ZJP group. D, H, L: Low-dose ZJP group. 

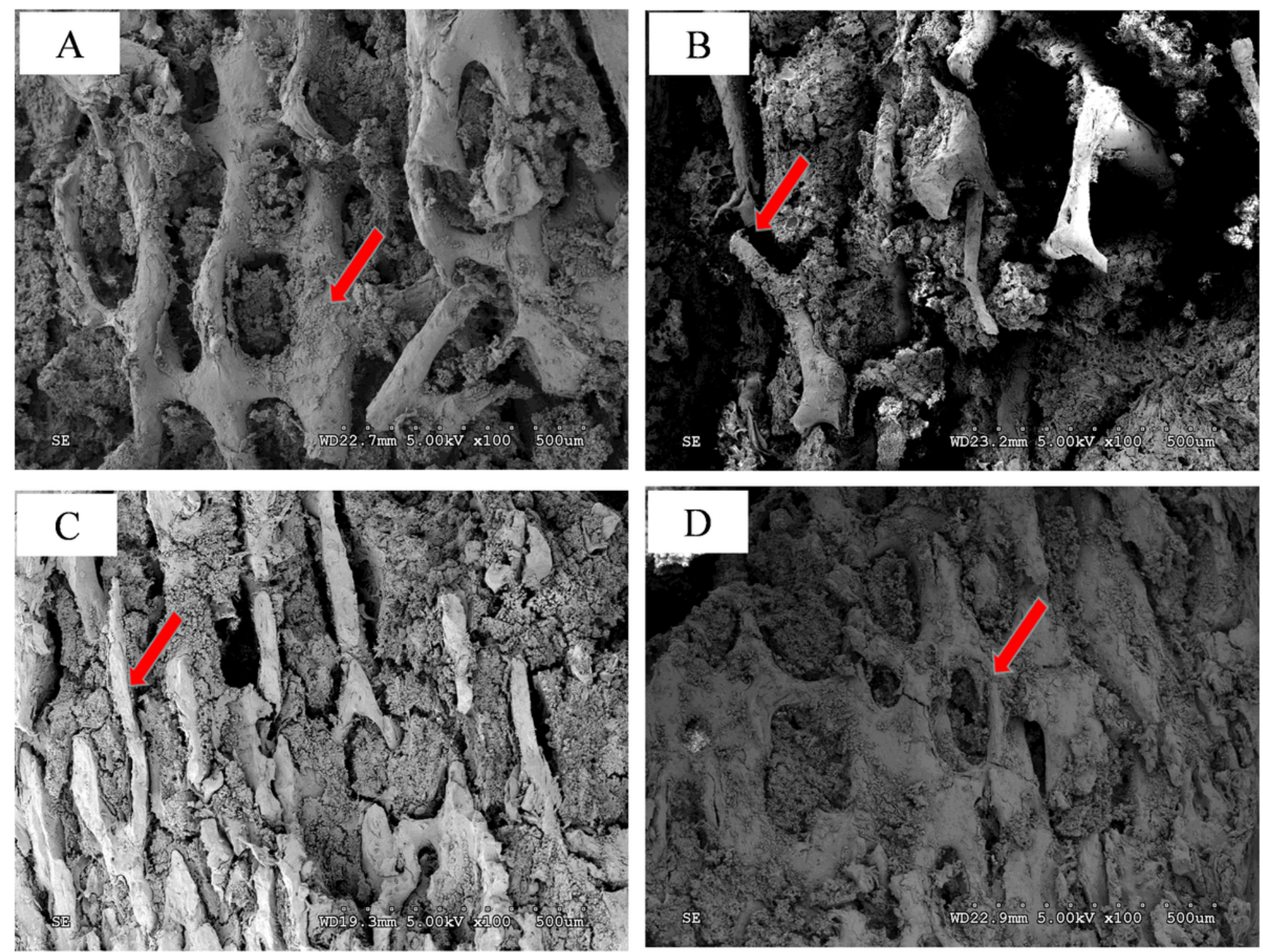

Figure 3

Scanning electron micrograph of a femur from each group $(\times 100)$. A. Normal group. B. Model group. C. High-dose ZJP group. D. Low-dose ZJP group. 
A

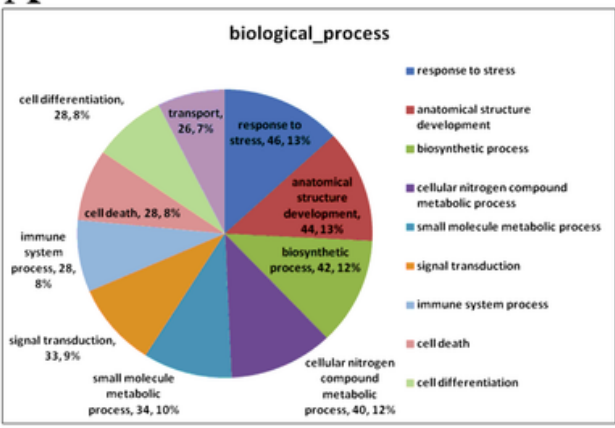

$\mathrm{D}$

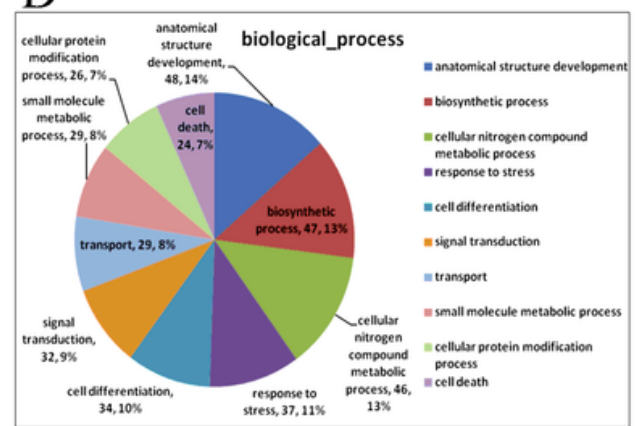

B

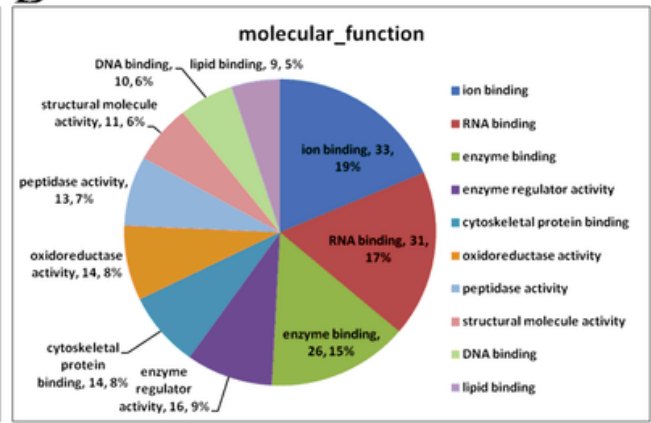

E

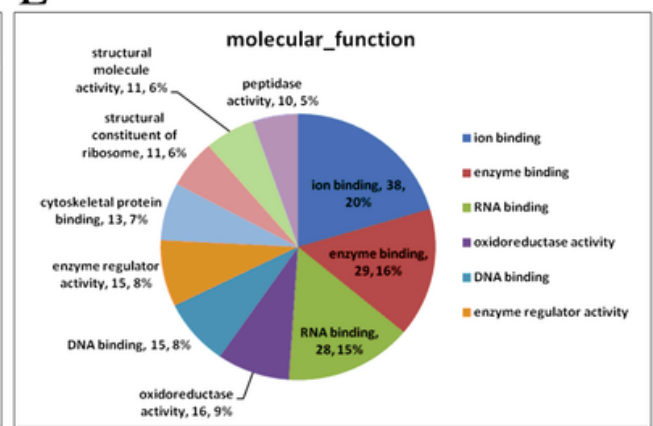

$\mathrm{C}$

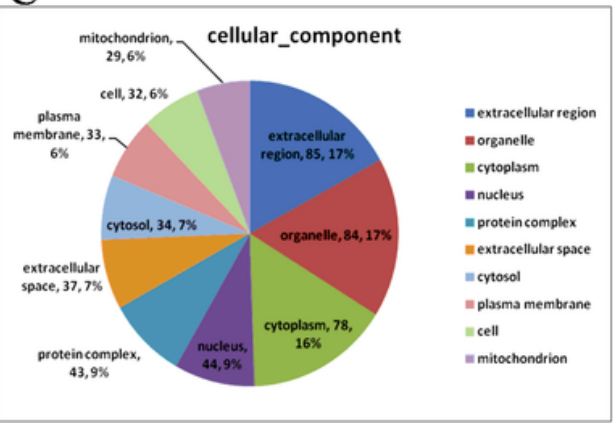

F

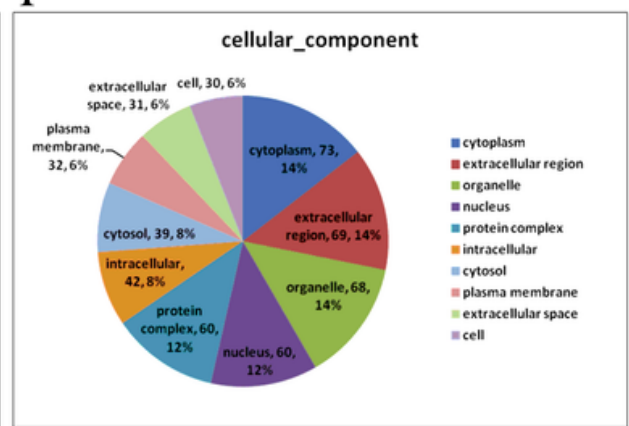

\section{Figure 4}

$\mathrm{GO}$ analysis results for the different groups of DOP rats. A. Biological process of the normal group vs. model group. B. Molecular function of the normal group vs. model group. C. Cellular component of the normal group vs. model group. D. Biological process of the model group vs. low-dose ZJP group. E. Molecular function of the model group vs. low-dose ZJP group. F. Cellular component of the model group vs. low-dose ZJP group.
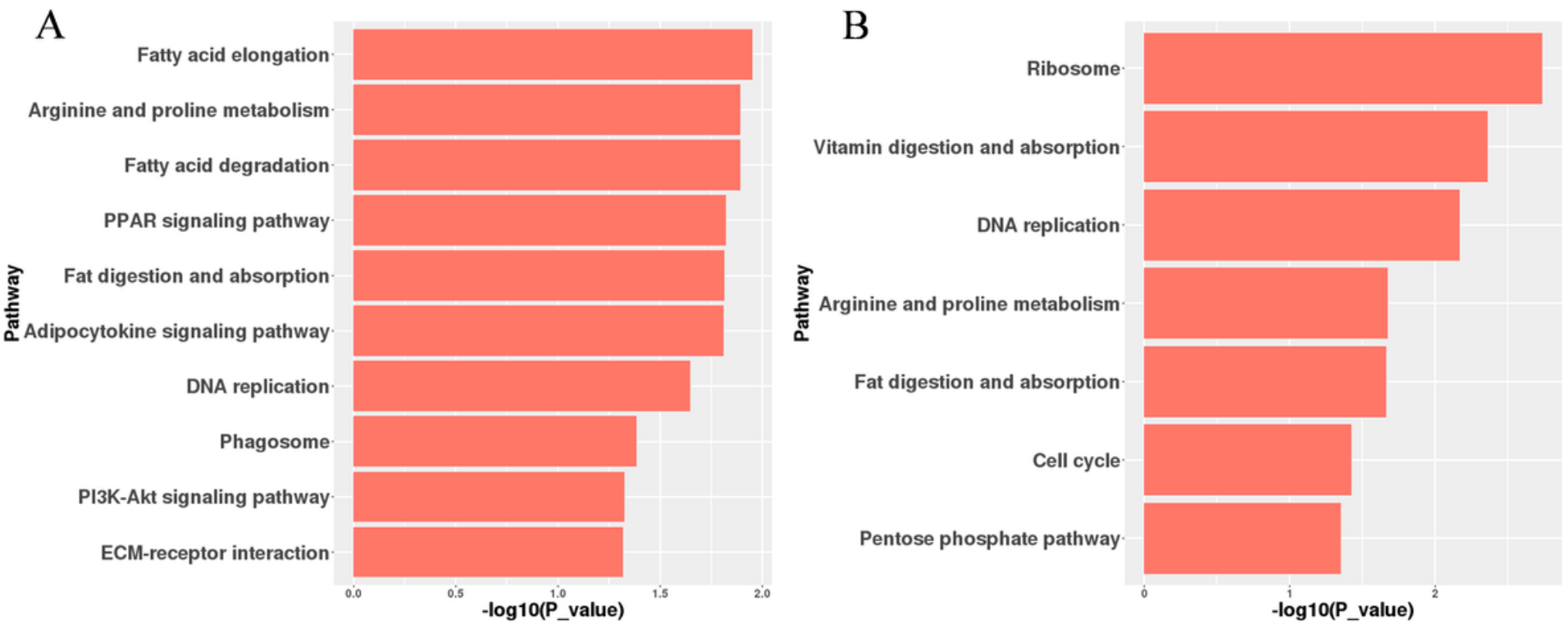

Figure 5 
Pathway analysis of the differentially expressed proteins between the different groups. A. Normal group vs. model group. B. Model group vs. low-dose ZJP group.

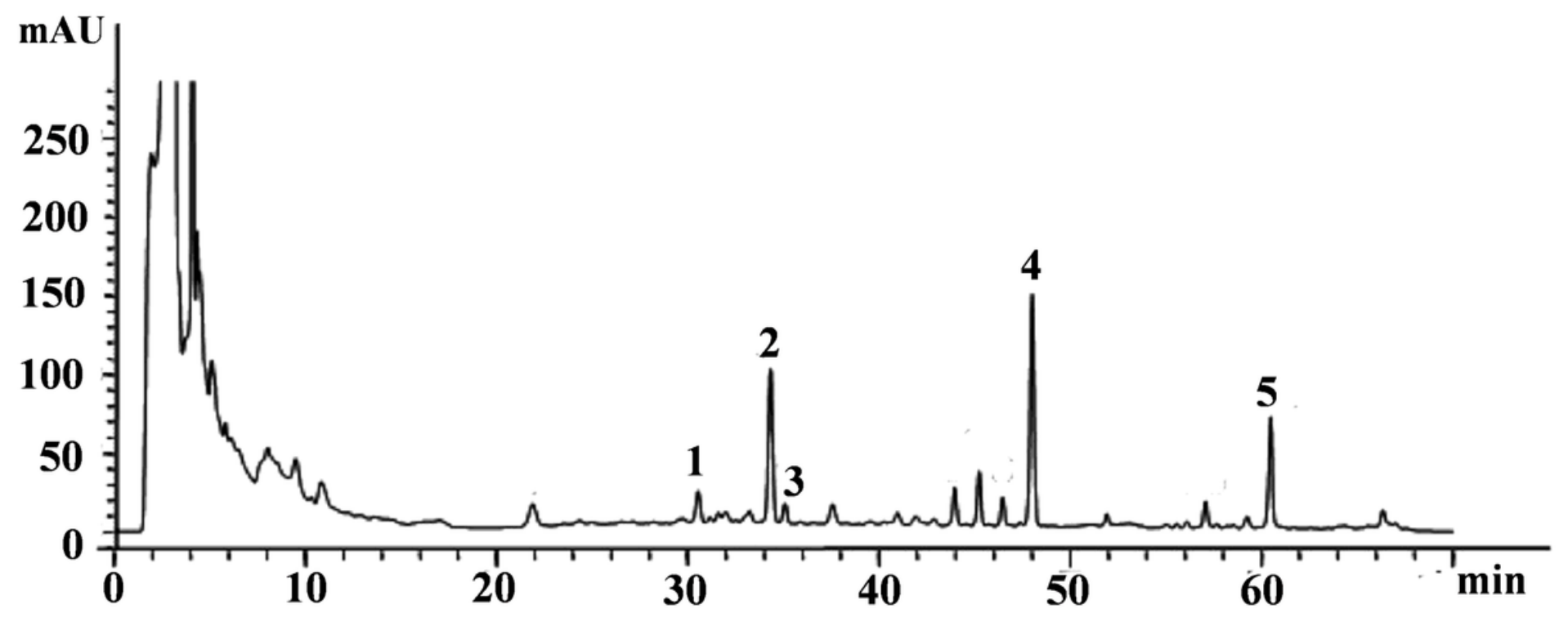

Figure 6

The HPLC fingerprint of ZJP. 